Chem. Ber. 118, $4377-4384$ (1985)

\title{
Tricarbonylchrom-Komplexe aromatischer Aldehyde und Ketone 1)
}

\author{
Franz Effenberger* und Klaus Schöllkopf ${ }^{2}$ ) \\ Institut für Organische Chemie der Universität Stuttgart, \\ Pfaffenwaldring 55, D-7000 Stuttgart 80
}

Eingegangen am 28. Januar 1985

Tricarbonylchrom (TCC)-Komplexe 2, 3 aromatischer Aldehyde und Ketone werden einmal durch basekatalysierte Carbodesilylierung von TCC-Aryltrimethylsilan-Komplexen 1 mit Dimethylformamid bzw. Benzoylfluorid, zum anderen durch selektive Oxidation von TCC-Phenylmethanol-Komplexen $\mathbf{5}$ mit speziell präpariertem Mangandioxid in Ether dargestellt.

\section{Arene Tricarbonylchromium Complexes of Aromatic Aldehydes and Ketones1)}

Arene tricarbonylchromium (TCC) complexes 2,3 of aromatic aldehydes and ketones are prepared by carbodesilylation of TCC aryltrimethylsilane complexes 1 with dimethylformamide and benzoyl fluoride, resp., or by selective oxidation of the corresponding benzyl alcohol TCC complexes 5 with especially prepared manganese dioxide in ether.

Die bislang beschriebenen Darstellungsmöglichkeiten von Tricarbonylchrom(TCC)-Komplexen aromatischer Aldehyde und Ketone sind unbefriedigend. Thre direkte Synthese aus entsprechenden Carbonylverbindungen mit Hexacarbonylchrom ist mit Aldehyden nicht ${ }^{3)}$ und mit Ketonen nur in schlechten Ausbeuten möglich ${ }^{4}$. Die TCC-Komplexe aromatischer Aldehyde werden deshalb in der Regel über die Komplexierung der entsprechenden Acetale und anschließende Hydrolyse ${ }^{3,5)}$ oder durch Reduktion der entsprechenden TCC-Benzoesäure-Komplexe mit komplexen Hydriden hergestellt ${ }^{6}$. . Bei der direkten Komplexierung von Benzophenonen, die in den beiden Ringen unterschiedlich substituiert sind, ist neben den geringen Ausbeuten die Bildung schlecht trennbarer Produktgemische von Nachteil 7). Da die direkte Acylierung der TCC-Aromaten-Komplexe in mäßigen Ausbeuten verläuft ${ }^{4)}$, werden die TCC-Komplexe von Benzophenonen mit Grignardverbindungen ${ }^{8 a)}$ oder Lithiumarylen ${ }^{8 b}$ ) hergestellt.

Aufgrund der in der vorstehenden Arbeit ${ }^{1}$ ) beschriebenen basekatalysierten Carbodesilylierung von TCC-Aryltrimethylsilan-Komplexen mit Aldehyden und Ketonen schienen uns zwei Wege zur Synthese von TCC-Komplexen aromatischer Aldehyde und Ketone möglich: einmal die direkte elektrophile Einführung einer Formyl- oder Acylgruppe durch basekatalysierte Carbodesilylierung von TCC-Aryltrimethylsilan-Komplexen und zum zweiten die selektive Oxidation der TCCkomplexierten Benzylalkohole bzw. Benzhydrole zu den entsprechenden Carbonylverbindungen unter Erhaltung des TCC-Liganden. Während der Durchführung unserer Arbeiten erschjen eine Publikation ${ }^{9)}$ über die selektive Oxidation TCC-komplexierter Benzylalkohole mit Dimethylsulfoxid/Acetanhydrid als Oxidationsmittel.

\section{Formylierung und Acylierung von TCC-Aryltrimethylsilan-Komplexen}

Die allgemein übliche elektrophile Einführung der Formylgruppe in Aromaten mit Ameisensäure-Derivaten ${ }^{10)}$ ist bei den desaktivierten TCC-Aromaten-Komplexen erschwert. Wir haben daher die basekatalysierte Carbodesilylierung der TCC-Phenyltri- 
methylsilan-Komplexe 1 in Gegenwart von Formylierungs- und Acylierungsagentien untersucht. Eine Formylierung des TCC-Phenyltrimethylsilan-Komplexes 1a gelang nicht mit Orthoameisensäure-triethylester oder Formylacetat, jedoch mit Dimethylformamid (DMF) unter Zugabe von Caesiumfluorid bei $60^{\circ} \mathrm{C}$, wobei DMF zugleich als Lösungsmittel diente; nach $6 \mathrm{~h}$ Reaktionszeit und wäßriger Aufarbeitung wurde der TCC-Benzaldehyd-Komplex 2 in 40proz. Ausbeute isoliert.<smiles>CN(C)C(=O)[PH2+][Mg]</smiles>

1a

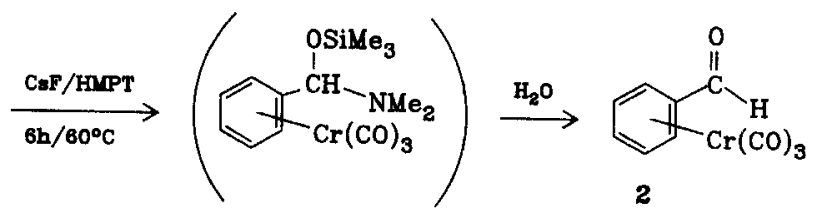

Längere Reaktionszeiten führten zu weitgehender Zersetzung, ein Folgeprodukt von 2 mit dem Substrat 1 a wurde nicht beobachtet.

Eine Benzoylierung der TCC-Komplexe 1a, b gelang uns mit Benzoylfluorid als dem gegenüber Phenylmagnesiumbromid reaktivsten Benzoylhalogenid ${ }^{11)}$ : In Gegenwart von CsF als Katalysator erhielten wir nach mehrstündigem Erwärmen in Hexamethylphosphorsäuretriamid (HMPT) die TCC-Benzophenon-Komplexe 3 in befriedigenden Ausbeuten.

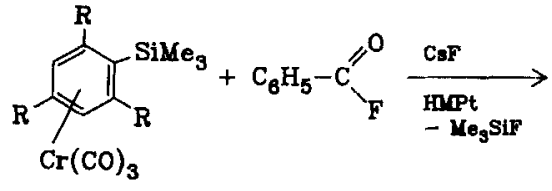

1a, $\mathbf{b}$<smiles>[R]c1cc([R])c(C([R])(C)C([R])([R])F)c([R])c1C(=O)c1ccccc1</smiles>

$$
\begin{aligned}
3 \mathbf{a}: \mathrm{R} & =\mathrm{H}(47 \%) \\
\mathbf{b}: \mathrm{R} & =\mathrm{CH}_{3}(72 \%)
\end{aligned}
$$<smiles>CC(C)(C)C(C)(C)C(C)(C)C(C)(C)C(C)(C)C(C)(C)C(C)(C)C(C)(C)C</smiles>

4

Die bei der Acylierung metallorganischer Verbindungen nicht zu vermeidende Weiterreaktion der entstehenden Ketone zu tertiären Alkoholen kann auch bei der Reaktion von 1 a mit Benzoylfluorid nicht vollständig unterdrückt werden, so daß - je nach Reaktionsbedingungen - neben 3a auch der TCC-Triphenylmethanol-Komplex 4 entsteht; bei dem sterisch gehinderten TCC-2,4,6-Trimethylbenzophenon-Komplex $3 \mathrm{~b}$ erfolgt diese Weiterreaktion erwartungsgemäß nicht.

Acylierungsversuche mit Benzoylchlorid anstelle von Benzoylfluorid gelangen nicht, auch nicht mit der doppelt molaren Menge CsF, die evtl. zu einem Halogenidaustausch hätte führen können. Größere CsF-Mengen beschleunigen die Zersetzung des Substrats.

\section{TCC-Komplexe aromatischer Ketone durch selektive Oxidation von TCC- komplexierten Benzhydrolen und Benzylalkoholen}

Die Oxidation von TCC-komplexierten primären oder sekundären Alkoholen zu TCC-KetonKomplexen scheitert mit üblichen Oxidationsmitteln im allgemeinen an der Empfindlichkeit der TCC-Gruppe gegenüber dem Oxidationsmittel. So wird z.B. der TCC-Benzylalkohol-Komplex mit Mangandioxid ausschließlich zu Benzaldehyd oxidiert ${ }^{4 a}$, dagegen gelingt die selektive Oxida- 
tion von TCC-Indanol- und TCC-Tetralol-Komplexen mit Mangandioxid zu den TCC-KetonKomplexen ${ }^{12)}$; in beiden Fällen findet man jedoch keine Angaben über die Darstellung des verwendeten Mangandioxids.

Die je nach Herstellung unterschiedliche Aktivität von Mangandioxid ist bekannt ${ }^{13)}$. Wir haben nun versucht, ein Mangandioxid zu erhalten, mit dem die vorstehend beschriebenen TCC-Benzhydryl-Komplexe $5^{1)}$ selektiv unter Erhaltung der TCC-Gruppe oxidiert werden können. Während mit käuflichem Mangandioxid keine Reaktion erfolgte, erhielten wir mit einem frisch gefällten aktivierten Produkt unter sofort einsetzender Gasentwicklung eine vollständige Oxidation des Tricarbonyl( $\alpha$-phenyl- $\eta^{6}$-benzolmethanol)chroms (5a) zu Benzophenon. Dieses frisch gefällte aktivierte Mangandioxid ,altert" im Laufe von 4 Wochen, verliert an Aktivität und führt dann zu einer Oxidation von 5a zu einem Gemisch aus Benzophenon und dem erwünschten TCCBenzophenon-Komplex 3a. Nach noch längerem Lagern wird dieses Mangandioxid gegenüber $\mathbf{5 a}$ völlig inaktiv, läßt sich jedoch durch Erwärmen i. Vak. so reaktivieren, daß es die TCC-Komplexe 5 selektiv und unter Erhaltung der TCC-Gruppe in guten Ausbeuten zu den TCC-Keton-Komplexen 3 oxidiert.

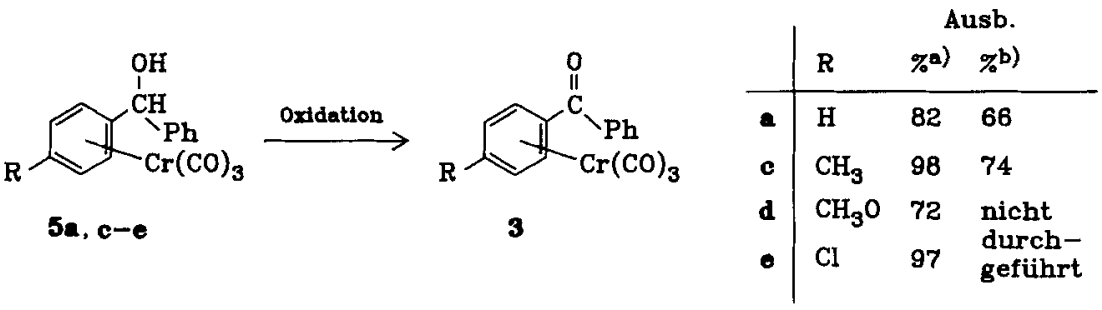

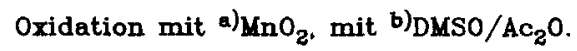

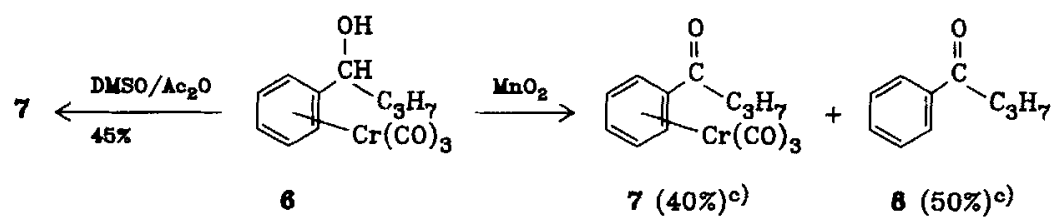

c) ${ }^{1} \mathrm{H}-\mathrm{NMR}-$ spektroskopisch bestimmt.

Bei der Oxidation des TCC-1-Phenylbutanol-Komplexes 6 mit demselben reaktivierten Mangandioxid erfolgte eine teilweise Dekomplexierung, das erhaltene Gemisch aus dem TCC-1-Phenylbutanon-Komplex 7 und 1-Phenylbutanon (8) konnte chromatographisch nicht getrennt werden. Dagegen gelang die selektive Oxidation von 6 mit Dimethylsulfoxid/Acetanhydrid ${ }^{9)}$ zu dem reinen TCC-1-Phenylbutanon-Komplex 7 in $45 \%$ Ausbeute. Daß sich auch die im nicht komplexierten Phenylrest substituierten TCCBenzhydrol-Komplexe 9 - deren Darstellung analog Lit. ${ }^{1)}$ aus 1 a und 4-substituierten Benzaldehyden erfolgt - zu den entsprechenden TCC-Keton-Komplexen oxidieren lassen, zeigt das Beispiel der Umsetzung von 9a mit Dimethylsulfoxid/Acetanhydrid zum 
TCC-Keton-Komplex 10a. Analog gelang die Oxidation des TCC-1,4-Phenylenbis(phenylmethanol)-Komplexes 11 zum TCC-Keton-Komplex 12.<smiles>[R]c1ccc(C(=O)c2ccc(C(=O)c3cccc(C(=O)c4ccc(C)cc4)c3)cc2)cc1</smiles>

1a

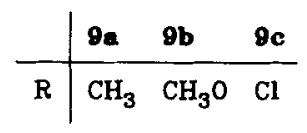

$10 a$<smiles>O=C(Cl)C(=O)c1cc(C(O)c2ccccc2)ccc1C(O)c1ccccc1</smiles>

11<smiles>O=C(c1ccccc1)c1ccc(C(=O)c2ccccc2)c(C(=O)OCc2ccccc2)c1</smiles>

12

Wir danken dem Fonds der Chemischen Industrie und der Deutschen Forschungsgemeinschaft für die Unterstützung der Arbeit. Für präparative Mitarbeit im Rahmen des Forschungspraktikums danken wir Frau cand. chem. S. Bihr und Herrn cand. chem. K. van Jan.

\section{Experimenteller Teil}

Gaschromatographie: Gerät 5700 der Fa. Packard mit Flammenionisationsdetektor (FID), Integrator: Spectraphysics Minigrator. $30 \mathrm{ml} / \mathrm{min}$ Reinstickstoff, Glaskapillare $1.8 \mathrm{bzw}, 2.30 \mathrm{~m} \times$ $2 \mathrm{~mm}$, OV $25 / 5 \%$ und OV $101 / 5 \%$ jeweils auf Gaschrom Q, $100-300^{\circ} \mathrm{C}, 16^{\circ} / \mathrm{min}$. - Präparative Gaschromatographie: Glassäulen verschiedener Dimensionen, Kieselgel 60, Korngröße $0.40-0.063 \mathrm{~mm}$ (Merck) oder Kieselgel S, 0.032-0.063 mm (Riedel-de Haën). Analytische Dünnschichtchromatographie: Polygram Sil G/UV Fertigfolien (Macherey \& Nagel). $-{ }^{1} \mathrm{H}$ NMR-Spektren: Geräte T 60, EM 360 der Fa. Varian sowie WP 80 und HX 90 der Fa. Bruker. ${ }^{13} \mathrm{C}-\mathrm{NMR}$-Spektren: HX $90(22.63 \mathrm{MHz})$ der Fa. Bruker. - IR-Spektren: Perkin-Elmer-Registrierphotometer 221 und $700 \mathrm{E}$.

Allgemeines: Sämtliche Reaktionen wurden unter trockenem Stickstoff durchgeführt. Die Lösungsmittel wurden gereinigt und wasserfrei eingesetzt. Diethylether wurde von Natriumbenzo-

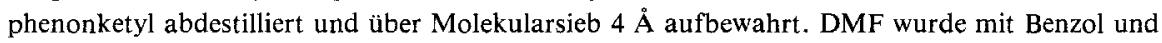
Wasser versetzt, dann wurde bei Normaldruck das azeotrope Gemisch abdestilliert und das so vorgereinigte DMF i. Wasserstrahlvak. destilliert. Das Destillat wurde bei $60^{\circ} \mathrm{C} 12 \mathrm{~h}$ mit Calciumhydrid gerührt, i. Vak. abdeștilliert und über Molekularsieb $3 \AA$ aufbewahrt. Hexamethylphosphorsäuretriamid (HMPT) wurde bei $80^{\circ} \mathrm{C} 36 \mathrm{~h}$ mit Calciumhydrid gerührt, i. Vak. abdestilliert und über Molekularsieb $4 \AA$ aufbewahrt.

Die Umsetzungen werden in einem Einhalskolben mit Magnetrührstäbchen durchgeführt. Zunächst wird CsF eingewogen, dann der Kolben insgesamt dreimal mit fächelnder Flamme ausgeheizt und nach dem Abkühlen mit Stickstoff belüftet. Anschließend wird das Substrat zugegeben, erneut dreimal evakuiert und mit Stickstoff belüftet. Unter Inertgas wird das Gasableitungsrohr mit Hahn gegen ein Gummiseptum (rubber septum, Fa. Aldrich) ausgetauscht, das Lösungsmittel 
(DMF oder HMPT) und anschließend das Elektrophil jeweils mit einer Spritze unter Rühren zugetropft.

(Benzaldehyd) tricarbonylchrom (2): Wie vorstehend beschrieben, werden $1.43 \mathrm{~g}(5.0 \mathrm{mmol})$ Tricarbonyl[trimethyl $\left(\eta^{6}\right.$-phenyl)silan]chrom $(1 \mathrm{a})^{1)}$ und $0.080 \mathrm{~g}(0.50 \mathrm{mmol}) \mathrm{CsF}$ in $10 \mathrm{ml}(130$ mmol) DMF $6 \mathrm{~h}$ bei $60^{\circ} \mathrm{C}$ umgesetzt, anschließend wird in Wasser aufgenommen, mit $1 \mathrm{ml}$ Essigsäure versetzt, mit Diethylether extrahiert, der Etherextract mit Wasser gewaschen und über Calciumchlorid getrocknet. Das nach Abdestillieren des Ethers zurückbleibende tiefrote Öl wird über

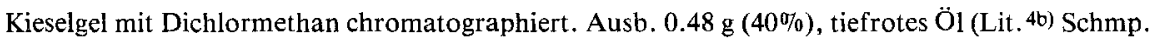
$\left.78.5-79.5^{\circ} \mathrm{C}\right) .-{ }^{1} \mathrm{H}-\mathrm{NMR}\left(\mathrm{CDCl}_{3}\right): \delta=9.5(\mathrm{~s}, 1 \mathrm{H}, \mathrm{CHO})\left[\right.$ Lit. $\left.{ }^{14)} \tau=5.25\right], 5.2-5.6(\mathrm{~m}, 5 \mathrm{H}$, Ph) [Lit. $\left.{ }^{14)} \tau=7.17\right] .-$ IR $\left(\mathrm{CDCl}_{3}\right): 1990,1870(\mathrm{C} \equiv \mathrm{O})$ [Lit. $\left.{ }^{4 b)} 1985,1910\right], 1690 \mathrm{~cm}^{-1}$ (CHO) [Lit.4b) $1700,2720,2857]$. ${ }^{13} \mathrm{C}-\mathrm{NMR}\left(\mathrm{CDCl}_{3}\right): \delta=229.89(\mathrm{C} \equiv \mathrm{O}), 187.67(\mathrm{CHO}) .-{ }^{1} \mathrm{H}-$ NMR- und IR-Spektren stimmen überein mit denen authentischer Substanz ${ }^{4 b)}$.

Umsetzungen von 1 a bzw. Tricarbonylltrimethyl( $\eta^{6}-2,4,6$-trimethylphenyl)silanjchrom (1 b) mit Benzoylfluorid

a) Von $1 \mathrm{a}: 1) 2.86 \mathrm{~g}(10 \mathrm{mmol}) 1 \mathrm{a}, 0.15 \mathrm{~g}$ (1.0 mmol) $\mathrm{CsF}$ und $1.24 \mathrm{~g}(10 \mathrm{mmol})$ Benzoylfluorid werden in $10 \mathrm{ml} \mathrm{HMPT}$ wie oben beschrieben $5 \mathrm{~h}$ bei $80^{\circ} \mathrm{C}$ umgesetzt. Nach dem Abkühlen wird das Reaktionsgemisch mit Diethylether über Kieselgel filtriert, die Etherlösung zweimal mit Wasser gewaschen, über Calciumchlorid getrocknet und der nach Abdestillieren des Ethers verbleibende Rückstand über Kieselgel mit Dichlormethan chromatographiert. Ausb. $1.5 \mathrm{~g}$ (47\%) Tricarbonyll( $\eta^{6}$-phenyl)phenylmethanonjchrom (3a) als zähes dunkelrotes Öl.

2) Wie unter a) werden jeweils $0.86 \mathrm{~g}$ (3.0 mmol) 1a mit $0.090 \mathrm{~g}(0.60 \mathrm{mmol}) \mathrm{CsF}$ und $0.74 \mathrm{~g}$ $(6.0 \mathrm{mmol})$ Benzoylfluorid unter Variation der Bedingungen umgesetzt und oxidativ aufgearbeitet. Hierzu wird nach beendeter Reaktion mittels einer Spritze eine Probe entnommen und zu einer gesättigten Iodlösung in Diethylether gegeben. Nach 20 min wird überschüssiges Iod mit wäßriger Natriumhydrogensulfit-Lösung reduziert, die Etherphase abgetrennt, mit Wasser gewaschen, über Magnesiumsulfat getrocknet und das Verhältnis Benzophenon/Triphenylmethanol gaschromatographisch bestimmt.

\begin{tabular}{|c|c|c|c|c|}
\hline \multirow[b]{2}{*}{ Nr. } & \multirow[b]{2}{*}{$\begin{array}{l}\text { Lösungsmittel } \\
\text { (ml) }\end{array}$} & \multicolumn{2}{|c|}{ Reaktions- } & \multirow{2}{*}{$\begin{array}{l}\text { Verhältnis (FID-\%) } \\
\text { Benzophenon/ } \\
\text { Triphenylmethanol }\end{array}$} \\
\hline & & Zeit & $\underset{{ }^{\circ} \mathrm{C}}{\text { Temp. }}$ & \\
\hline 1 & HMPT (1) & 0.1 & 100 & 2.5 \\
\hline 2 & HMPT (5) & 20 & 20 & 3.4 \\
\hline 3 & DMF (3) & 17 & 40 & 1.6 \\
\hline 4 & HMPT (5) & 1 & 80 & 4.8 \\
\hline
\end{tabular}

b) Von $1 \mathrm{~b}$ : Wie unter $\mathrm{a}, 1)$ beschrieben, werden $0.38 \mathrm{~g}(1.2 \mathrm{mmol}) \mathbf{1 b}, 0.030 \mathrm{~g}(0.20 \mathrm{mmol}) \mathrm{CsF}$ und $0.25 \mathrm{~g}(2.0 \mathrm{mmol})$ Benzoylfluorid in $5 \mathrm{ml} \mathrm{DMF} 4 \mathrm{~h}$ auf $50^{\circ} \mathrm{C}$ erwärmt. Anschließend wird das Reaktionsgemisch auf verd. Kalilauge gegossen, intensiv verrührt und zweimal ausgeethert. Die wäßrige Phase wird mit Eisessig neutralisiert und nochmals ausgeethert, die vereinigten Etherextrakte werden mit Wasser gewaschen und über Calciumchlorid getrocknet. Nach Abdestillieren des Ethers wird der feste Rückstand aus $n$-Hexan/Diethylether (2:1) umkristallisiert. Ausb. $0.30 \mathrm{~g}(72 \%)$ Tricarbonyllphenyl $\left(\eta^{6}-2,4,6\right.$-trimethylphenyl)methanon]chrom (3b), Schmp. ab $140^{\circ} \mathrm{C}$ (Zers.). $-{ }^{1} \mathrm{H}-\mathrm{NMR}\left(\mathrm{CDCl}_{3}\right): \delta=7.5-8.0(\mathrm{~m}, 5 \mathrm{H}, \mathrm{Ph}), 4.9(\mathrm{~s}, 2 \mathrm{H}, \mathrm{Ph}), 2.3(\mathrm{~s}, 3 \mathrm{H}$, 4- $\left.\mathrm{CH}_{3}\right), 2.1\left(\mathrm{~s}, 6 \mathrm{H}, 2,6-\mathrm{CH}_{3}\right)$. - IR $\left(\mathrm{CDCl}_{3}\right): 1970,1890(\mathrm{C} \equiv \mathrm{O}), 1670 \mathrm{~cm}^{-1}(\mathrm{C}=\mathrm{O})$.

$$
\mathrm{C}_{19} \mathrm{H}_{16} \mathrm{CrO}_{4} \text { (360.4) Ber. C } 63.32 \mathrm{H} 4.47 \text { Gef. C } 63.55 \mathrm{H} 4.64
$$

TCC-komplexierte Alkohole 9a-c nach Lit. ${ }^{1)}$ : Der Komplex $\mathbf{1 a}^{1{ }^{1)}}$ wird mit den zweimal destillierten Aldehyden wie unter Lit.1) beschrieben $3-4 \mathrm{~h}$ bei $20^{\circ} \mathrm{C}$ gerührt. Anschließend wird mit 
Wasser versetzt und weitere $2 \mathrm{~h}$ bei $20^{\circ} \mathrm{C}$ gerührt. Nach Stehenlassen über Nacht werden $150 \mathrm{ml}$ Ether zugegeben, die Lösung wird dann vom Unlöslichen abfiltriert, zweimal mit Wasser gewaschen und über Natriumsulfat getrocknet. Nach Entfernen des Lösungsmittels im Rotationsverdampfer wird das Rohprodukt an der Ölpumpe getrocknet und über eine Kieselgelsäule mit Dichlormethan chromatographiert oder aus Hexan/Toluol (4:1) umkristallisiert.

Tricarbonyl[4-methyl- $\alpha-1 \eta^{6}$-phenyl)benzolmethanol]chrom (9a): Aus $2.86 \mathrm{~g}$ (10 mmol) 1a, $1.3 \mathrm{~g}(10.8 \mathrm{mmol})$ 4-Methylbenzaldehyd und $0.15 \mathrm{~g}$ (1.0 mmol) $\mathrm{CsF}$ in $7.5 \mathrm{ml} \mathrm{DMF}$ und $0.25 \mathrm{ml}$ Wasser. Ausb. nach Chromatographie $1.7 \mathrm{~g} \mathrm{(50 \% )}$ gelbes Produkt, Schmp. $88-89^{\circ} \mathrm{C}$.

$$
\mathrm{C}_{17} \mathrm{H}_{14} \mathrm{CrO}_{4} \text { (334.3) Ber. C } 61.08 \mathrm{H} 4.22 \text { Gef. C } 61.19 \mathrm{H} 4.16
$$

Tricarbonyl[4-methoxy- $\alpha-\left(\eta^{6}\right.$-phenyl)benzolmethanol]chrom $(9 \mathrm{~b})$ : Aus $2.86 \mathrm{~g}(10 \mathrm{mmol}) 1 \mathrm{a}$, $1.47 \mathrm{~g}(10.8 \mathrm{mmol})$ 4-Methoxybenzaldehyd und $0.15 \mathrm{~g}$ (1.0 mmol) CsF in $7.5 \mathrm{ml}$ DMF und $0.25 \mathrm{ml}$ Wasser. Ausb. nach zweimaliger Chromatographie und anschließendem Umkristallisieren $1.5 \mathrm{~g}$ (43\%) gelbes Produkt, Schmp. $86^{\circ} \mathrm{C}$.

$$
\mathrm{C}_{17} \mathrm{H}_{14} \mathrm{CrO}_{5} \text { (350.3) Ber. C } 58.29 \mathrm{H} 4.02 \text { Gef. C } 58.13 \mathrm{H} 4.01
$$

Tricarbonyl[4-chlor- $\alpha-\left(\eta^{6}\right.$-phenyl)benzolmethanol]chrom (9c): Aus $2.86 \mathrm{~g}(10 \mathrm{mmol}) 1 \mathrm{a}, 1.42 \mathrm{~g}$ (10 mmol) 4-Chlorbenzaldehyd und $0.15 \mathrm{~g}(1.0 \mathrm{mmol}) \mathrm{CsF}$ in $7.5 \mathrm{ml} \mathrm{DMF}$ und $0.25 \mathrm{ml}$ Wasser. Ausb. nach Chromatographie und anschließendem Umkristallisieren $2.6 \mathrm{~g}(73 \%)$ gelbes Produkt, Schmp. $102^{\circ} \mathrm{C}$.

$$
\mathrm{C}_{16} \mathrm{H}_{11} \mathrm{ClCrO}_{4} \text { (354.7) Ber. C } 54.17 \mathrm{H} 3.12 \mathrm{Cl} 9.99 \text { Gef. C } 54.27 \mathrm{H} 3.07 \mathrm{Cl} 10.01
$$

Selektive Oxidation der TCC-komplexierten Alkohole 5, 9a und 11 mit Mangandioxid bzw. mit Dimethylsulfoxid/Acetanhydrid

a) Mangandioxid nach Lit. 15$)$ : Zur Lösung von $17.4 \mathrm{~g}(0.11 \mathrm{~mol})$ Kaliumpermanganat in $75 \mathrm{ml}$ Wasser gibt man zunächst portionsweise $50 \mathrm{ml}$ Ethanol, wobei starke Erwärmung erfolgt. Nach $1 \mathrm{~h}$ werden $200 \mathrm{ml}$ kaltes Wasser zugegeben, dann wird das Gemisch kräftig aufgeschlämmt, das ausgefallene Mangandioxid abfiltriert, der Filterkuchen mehrmals mit Wasser gewaschen und bei $80^{\circ} \mathrm{C}$ getrocknet. Nach 6-8wöchigem Stehenlassen wird das Mangandioxid $2-3 \mathrm{~h}$ bei ca. $10^{-2}$ Torr auf $80^{\circ} \mathrm{C}$ erwärmt. Die Aktivität des jeweils eingesetzten Mangandioxids wird vor jeder Umsetzung bestimmt, wozu zu der Lösung von $0.010 \mathrm{~g} 3 \mathrm{a}$ in $2 \mathrm{ml}$ Diethylether portionsweise Mangandioxid gegeben wird und die Reaktion mittels Dünnschichtchromatographie mit Dichlormethan als Laufmittel verfolgt wird.

b) Oxidation der Alkohole mit Mangandioxid: Die Komplexe 5 werden mit dem vorstehend beschriebenen Mangandioxid in Diethylether unter Stickstoff bei $20^{\circ} \mathrm{C}$ gerührt, anschließend wird vom Unlöslichen abfiltriert und der Ether abdestilliert.

c) Oxidation der Alkohole mit Dimethylsulfoxid (DMSO)/Acetanhydrid nach Lit. 9): Die TCCAlkohol-Komplexe läßt man mit DMSO und Acetanhydrid in einem 50-ml-Kolben die angegebene Zeit bei Raumtemp. im Dunkeln stehen, wobei sich die Reaktionsgemische rot färben. Nach Zugeben von $60 \mathrm{ml}$ Toluol wird mit 20proz. Natronlauge neutralisiert, fünfmal mit Wasser gewaschen, das Toluol im Rotationsverdampfer entfernt, das rote Rohprodukt an der Ölpumpe getrocknet und anschließend über eine Kieselgelsäule mit Dichlormethan chromatographiert. Die reinen Substanzen (rote Feststoffe oder Öle, die allmählich fest werden) werden an der Ölpumpe getrocknet.

\section{Tricarbonyll $\left(\eta^{6}\right.$-phenyl)phenylmethanonjchrom (3a)}

$N a c h$ b): Aus $0.50 \mathrm{~g}(1.0 \mathrm{mmol})$ Tricarbonyl $\left(\alpha-\right.$ phenyl $-\eta^{6}$-benzolmethanol)chrom $(5 \mathrm{a})^{1)}$ und $0.64 \mathrm{~g}$ Mangandioxid in $30 \mathrm{ml}$ Diethylether nach $20 \mathrm{~h}$ Ausb. $0.42 \mathrm{~g} \mathrm{(82 \% )} \mathrm{3a} \mathrm{als} \mathrm{zähes} \mathrm{dunkelrotes}$ Öl (Lit. ${ }^{6 b)}$ orangerote Nadeln), Schmp. $88-89^{\circ} \mathrm{C}$ (Petrolether/Benzol). ${ }^{1} \mathrm{H}-\mathrm{NMR}$ - und IR-spektroskopisch identisch mit authentischer Substanz ${ }^{6 b)}$. 
Nach c): Aus $0.31 \mathrm{~g}(1.0 \mathrm{mmol}) 5 \mathrm{a}, 15 \mathrm{ml}$ DMSO und $10 \mathrm{ml} \mathrm{Ac} \mathrm{C}_{2} \mathrm{O}$ nach 90 min Reaktionszeit und Aufarbeiten Ausb. $0.20 \mathrm{~g}(66 \%)$ rotes Festprodukt, Schmp. $86^{\circ} \mathrm{C}$.

Tricarbonyll $\left(\eta^{6}-4-m e t h y l p h e n y l\right) p h e n y l m e t h a n o n j c h r o m(3 \mathrm{c}): \mathrm{Nach}$ b) aus $0.50 \mathrm{~g}(1.5 \mathrm{mmol})$ Tricarbonyl(4-methyl- $\alpha$-phenyl- $\eta^{6}$-benzolmethanol)chrom (5c) ${ }^{1)}$ und $1.0 \mathrm{~g}$ Mangandioxid in $30 \mathrm{ml}$ Diethylether nach $12 \mathrm{~h}$ Ausb, $0.49 \mathrm{~g}(98 \%)$ rotes Festprodukt, Schmp. $118-120^{\circ} \mathrm{C}$ (Lit. ${ }^{16)}$ $\left.122-123^{\circ} \mathrm{C}\right)$.

Nach c) aus $0.39 \mathrm{~g}(1.16 \mathrm{mmol}) 5 \mathrm{c}, 17.5 \mathrm{ml}$ DMSO und $11.6 \mathrm{ml} \mathrm{Ac} \mathrm{O}_{2} \mathrm{O}$ nach $1.75 \mathrm{~h}$ Reaktionszeit und Aufarbeiten Ausb. $0.30 \mathrm{~g} \mathrm{(74 \% )}$ rotes Öl.

Tricarbonyll( $\eta^{6}-4-$ methoxyphenyl)phenylmethanonjchrom (3d): Nach b) aus $0.50 \mathrm{~g}(1.4 \mathrm{mmol})$ Tricarbonyl(4-methoxy- $\alpha$-phenyl- $\eta^{6}$-benzolmethanol)chrom (5d) ${ }^{1)}$ und $1.0 \mathrm{~g}$ Mangandioxid in $30 \mathrm{ml}$ Diethylether wird das nach $12 \mathrm{~h}$ Reaktionszeit und nach Aufarbeiten erhaltene Produkt über Kieselgel mit Dichlormethan chromatographiert. Ausb. $0.35 \mathrm{~g}(72 \%)$ rotes Festprodukt, Schmp. $136-138^{\circ} \mathrm{C}$ (Lit. $\left.8 \mathrm{cc}\right) 140^{\circ} \mathrm{C}$ ).

Tricarbonyll( $\eta^{6}-4$-chlorphenyl)phenylmethanon/chrom $(3 \mathrm{e})$ : Nach b) aus $0.50 \mathrm{~g}(1.4 \mathrm{mmol})$ Tricarbonyl(4-chlor- $\alpha$-phenyl- $\eta^{6}$-benzolmethanol)chrom (5e) ${ }^{1)}$ und $1.0 \mathrm{~g}$ Mangandioxid in $30 \mathrm{ml}$ Diethylether wie bei $3 \mathrm{c}$ beschrieben. Ausb. $0.48 \mathrm{~g}(97 \%)$ rotes Festprodukt, Schmp. $79-81^{\circ} \mathrm{C}$. ${ }^{1} \mathrm{H}-\mathrm{NMR}\left(\mathrm{CDCl}_{3}\right): \delta=7.4-7.9(\mathrm{~m}, 5 \mathrm{H}, \mathrm{Ph}), 5.6 \mathrm{bzw} .6 .2$ (2d, 4H, AA'BB'-System, Ph). - IR $\left(\mathrm{CDCl}_{3}\right): 1990,1930(\mathrm{C} \equiv \mathrm{O}), 1650 \mathrm{~cm}^{-1}(\mathrm{C}=\mathrm{O})$.

\section{$\mathrm{C}_{16} \mathrm{H}_{19} \mathrm{ClCrO}_{4}(352.7) \quad$ Ber. C $55.47 \mathrm{H} 2.57 \mathrm{Cl} 10.04$ Gef. C $54.49 \mathrm{H} 2.55 \mathrm{Cl} 10.29$}

\section{Tricarbonyll1-( $\eta^{6}$-phenyl)butanon]chrom (7)}

Nach b): $\mathrm{Zu}$ der Lösung von $0.50 \mathrm{~g}(1.7 \mathrm{mmol})$ Tricarbonyl( $\alpha$-propyl- $\eta^{6}$-benzolmethanol)chrom (6)1) in $40 \mathrm{ml}$ Diethylether wird portionsweise $\mathrm{MnO}_{2}$ gegeben, wobei der Reaktionsablauf dünnschichtchromatographisch verfolgt wurde. Nach $30 \mathrm{~min}$ war vollständige Reaktion eingetreten (nach Zugabe von $5.0 \mathrm{~g} \mathrm{MnO}_{2}$ ). Ausb. nach Abziehen des Ethers über eine G3-Fritte $0.47 \mathrm{~g}$ (Rohprodukt), das nach gaschromatographischer Untersuchung aus $40 \% 7$ und 50\% Propylphenylketon (8) bestand.

Nach c): Aus $0.29 \mathrm{~g}$ (1.0 mmol) 6, $15 \mathrm{ml}$ DMSO und $10 \mathrm{ml} \mathrm{Ac} 2 \mathrm{O}$ nach $1.75 \mathrm{~h}$ Reaktionsdauer und Aufarbeiten Ausb. $0.13 \mathrm{~g} \mathrm{(45 \% )}$ rotes Öl.

$$
\mathrm{C}_{13} \mathrm{H}_{12} \mathrm{CrO}_{4} \text { (284.2) Ber. C } 54.94 \mathrm{H} 4.26 \text { Gef. C } 55.25 \mathrm{H} 4.81
$$

Tricarbonyl(4-methylphenyl)- $\eta^{6}$-phenylmethanon/chrom (10a): $\mathrm{Nach} \mathrm{c}$ ) aus $0.33 \mathrm{~g}(1.0 \mathrm{mmol})$ 9a, $15 \mathrm{ml} \mathrm{DMSO}$ und $10 \mathrm{ml} \mathrm{Ac} \mathrm{A}_{2} \mathrm{O}$ nach $3.5 \mathrm{~h}$ Reaktionsdauer und Aufarbeiten; Ausb. $0.28 \mathrm{~g}$ $(85 \%)$ rotes ÖI.

$$
\mathrm{C}_{17} \mathrm{H}_{12} \mathrm{CrO}_{4} \text { (332.3) Ber. C } 61.43 \mathrm{H} 3.64 \text { Gef. C } 61.35 \mathrm{H} 4.40
$$

Tricarbonyl(1,4-dibenzoyl- $\eta^{6}$-benzol/chrom (12): Nach c) aus $0.42 \mathrm{~g}(1.0 \mathrm{mmol})$ Tricarbonyl( $\alpha, \alpha^{\prime}$-diphenyl- $\eta^{6}$-1,4-benzoldimethanol)chrom ${ }^{1}$ ), $30 \mathrm{ml}$ DMSO und $20 \mathrm{ml} \mathrm{Ac} 2 \mathrm{O}$ nach $3 \mathrm{~h}$ Reaktionsdauer und Aufarbeiten; Ausb. $0.35 \mathrm{~g}(83 \%)$ rotes Öl.

$$
\mathrm{C}_{23} \mathrm{H}_{14} \mathrm{CrO}_{5} \text { (422.3) Ber. C } 65.40 \mathrm{H} 3.34 \text { Gef, C } 65.30 \mathrm{H} 4.15
$$

1) 30. Mitteil. zur elektrophilen Aromatensubstitution; 29. Mitteil.: F. Effenberger und $K$. Schöllkopf, Chem. Ber. 118, 4356 (1985), vorstehend.

2) $K$. Schöllkopf, Teil der Dissertation, Univ. Stuttgart 1982.

3) $R$. Dabard, P. Fournari und J. Besancon, C. R. Acad. Sci. 260, 2833 (1965),

4) 4a) E. Mostardini, F. Calderazzo und R. Ercoli, Chim. Ind. (Milano) 42, 1231 (1960). 4b) G. Drefahl, H.-H. Hörhold und K. Kühne, Chem. Ber. 98, $1826(1965) .-4 c)$ A. SolladieCavallo, G. Solladie und E. Tsamo, J. Org. Chem. 44, 4189 (1979). 
5) R. Dabard und G. Jaouen, Tetrahedron Lett. 1969, 3391 ,

6) 6a) B. Nicholls und $M$. C. Whiting, J. Chem. Soc. 1959, 551. - 6b) J. D. Holmes, D. A. K. Jones und R. Pettit, J. Organomet. Chem. 4, 324 (1965).

7) 7a) S. Top und G. Jaouen, J. Organomet. Chem. 182, 381 (1979). - 7b) N. J. Gogan, I. L. Dickinson und $J$. R. Patterson, J. Organomet. Chem. 214, 65 (1981).

8) 8a) J. Besancon, J. Tirouflet, A. Card und $Y$. Dusausoy, J. Organomet. Chem. 59, 267 (1973). - 8b) A. Meyer, Ann. Chim. Ser. 14, 8, 315 (1973).

9) $S$. G. Levine und B. Gopalakrishnan, Tetrahedron Lett. 1982, 1239.

10) G. Simchen in Methoden der Organischen Chemie (Houben-Weyl-Müller), 4. Aufl., Bd. E 3 , S. $16 \mathrm{ff}$., Georg Thieme Verlag Stuttgart - New York 1983.

11) C. E. Entenmann und J. R. Johnson, J. Am. Chem. Soc. 55, 2900 (1933).

12) G. Jaouen und A. Meyer, J. Am. Chem. Soc. 97, 4667 (1975).

13) A. J. Fatiadi, Synthesis 1976, 65.

14) M. D. Rausch, G. A. Moser, E. J. Zaiko und A. L. Lipman, J. Organomet. Chem. 23, 185 (1970).

15) J. J. Eisch und R. B. King, Organometallic Syntheses, Vol. 1, S. 96, Academic Press, New York 1965.

16) N. J. Gogan, I. L. Dickinson und J. R. Patterson, J. Organomet. Chem. 214, 65 (1981).

$[13 / 85]$ 